\title{
MICROSCOPIC CALCULATION OF THE NUCLEAR MEAN FIELD(1)
}

\author{
J. CUGNON, P. GRANGE* and A. LEJEUNE \\ Université de Liège, Physique Nucléaire Théorique, Institut \\ de Physique au Sart Tilman, B5, B-4000 Liege 1 ., Belgium \\ - Centre de Recherches Nucléaires, BP 20 CRO, \\ F-67037 Strasbourg Cedex, France
}

\begin{abstract}
Résumé - Pour un système de matière nucléaire à densité normale, nous étudions la contribution, au champ nucléaire moyen, des corrélations de l'état fondamental et ce, pour des températures nulle et finie. Nous travaillons dans le cadre d'un développement en nombre de lignes trou en utilisant le potentiel nucléon-nucléon de Paris. Nous mettons en exergue la relation existante avec le terme de collision de l'équation de Landau-Vlassov.

Abstract - Zero and finite temperature contribution of ground state correlations to the nuclear mean field is studied for nuclear matter at normal density within the framework of the hole line expansion using the Paris potential. The connection with the collision term of the Landau-Vlassov equation is exhibited.
\end{abstract}

\section{INTRODUCTION}

The knowledge of the nuclear mean field is very important to explain the static as well as dynamic properties of nuclear systems. It is then highly desirable to understand the properties of this mean field from first principles. By this term, we here mean nuclear forces described by potentials in the frame of nonrelativistic quantum mechanics. A very intensive effort has been devoted to the ground state nuclear matter mean field in the past. But, despite of this effort, the mean field is known in first order only, not in the bare interaction, but in the renormalized interaction which accounts for medium effects. Here, we present the first microscopic calculation, based on a realistic NN interaction (namely the Paris potential), which goes up to second order in the renormalized interaction and so takes the ground state correlations into account. The calculation is done for zero and finite temperatures.

\section{THE FORMAL ISM}

The framework is the Brueckner approach to nuclear matter (see ref. [1] for a review), extended to finite temperature according to the method of ref. [2]. The Green function, which describes at the same time the propagation of a particle added to the medium and the one of a hole punched into the medium (of momentum $\vec{k}$ and energy $E)$, can be written as $(K=1)$

$$
G(k, E)=\frac{1}{E-\frac{k^{2}}{2 m}-M(k, E)} \text {. }
$$

The quantity $M(k, E)$, known as the mass operator, can be expanded in series of the Brueckner reaction matrix $g$. The latter is related to the bare NN potential $V$

(1) Supported by the NATO grant $\mathrm{N}^{\circ} 025.81$ 
through the Bethe-Goldstone equation

$$
g(\omega)=v+v \frac{Q}{\omega-e(a)-e(b)} g(\omega),
$$

$\mathrm{Q}$ being the Pauli operator for the intermediate states. Up to second order in $g$, one has :

$$
M(k, E)=M_{1}(k, E)+M_{2}(k, E),
$$

with

$$
\begin{gathered}
M_{1}(k, E)=\sum_{\vec{j}} n(j)\langle\overrightarrow{k j}|g[E+e(j)]| \widetilde{\vec{k}}\rangle, \\
M_{2}(k, E)=\frac{1}{2} \sum_{\vec{j} \vec{j} \vec{a}} n(j) n(l)(1-n(a)) \frac{|\langle\vec{j} \vec{l} \mid g[e(j)+e(l)] \widetilde{\overrightarrow{k a}}\rangle|^{2}}{E+e(a)-e(j)-e(l)-i \varepsilon},
\end{gathered}
$$

where $e(k)$ is the single-particle energy for state $k$. It has to be determined self-consistently as

$$
e(k)=\frac{k^{2}}{2 m}+\operatorname{Re} M(k, e(k)) .
$$

In this scheme,

$$
M(k, e(k))=V(k)+i W(k),
$$

represents the momentum dependent complex mean field in nuclear matter (up to second order), identified to the optical-model potential. Eqs. (2.2)-(2.4) correspond to the diagrams contained in Fig. 1. The diagram on the left is called pola-

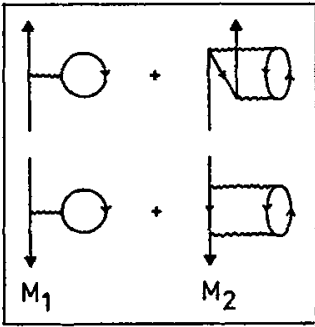

First order contributions to particle (upper row) and to hole (lower row) self-energy.

[Fig. 1] risation diagram and the other one the correlation diagram. These denominations remind of the physical meaning of the diagrams for particle states. The first one describes the reaction of the medium on the propagation of a particle due to the polarisation produced by this particle. The correlation diagram describes the effect of the correlations present in the medium prior to the interaction of the particle. In Eqs. $(2.3)-(2.7)$, the quantities $n(k)$ are the occupation probabilities in the unperturbed ground state

$$
n(k)=\left\{1+\exp \left[\frac{e(k)-\mu}{T}\right]\right\}^{-1},
$$

where $\mu$ and the temperature $T$ are related to the nucleon density $\rho$ by

$$
\rho=\sum_{\vec{k}} n(k) \text {. }
$$

\section{THE MEAN FIELD}

In Fig. 2, we present our results for the real part of the correlation and the polarisation potentials for normal density $\rho_{0}=0.17 \mathrm{fm}^{-3}$. We also present $V_{\mathrm{po}}^{(0)}$, the mean field calculated in the first order approximation. We see that the polarisation field deepens when going to second order. However, the addition of the repulsive correlation field makes that globally, the mean field is less attractive than in first order approximation. This is an interesting result, which brings a better agreement with experiment, provided the deeplying single-particle states inside heavy nuclei can be considered as indicative of nuclear matter properties.

In Fig. 3, we show our results for finite temperature. Both the polarisation and the correlation fields decrease as the matter is heated up, but the total potential does present a weak temperature dependence. 


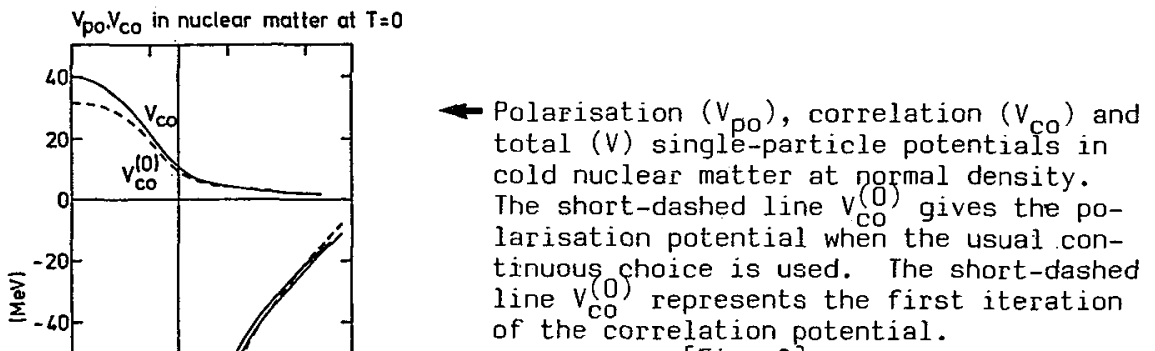

[Fig. 2]

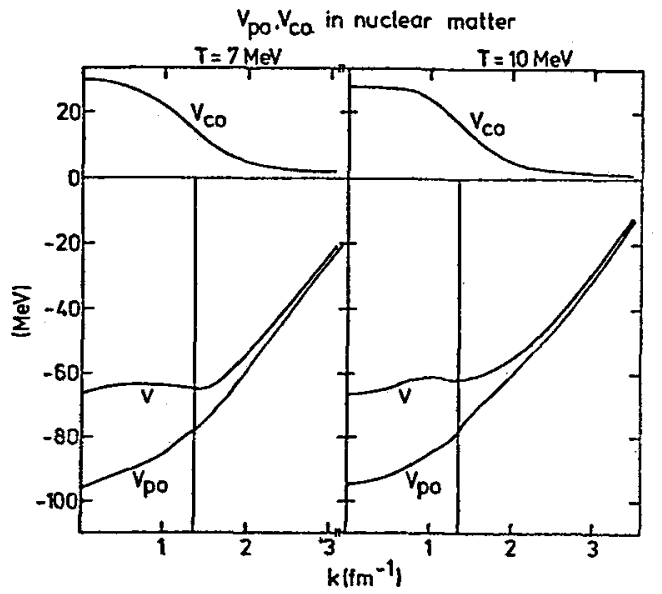

represents the true inertia of a particle embedded in the medium. It is given in Fig. 4 for various temperatures. The

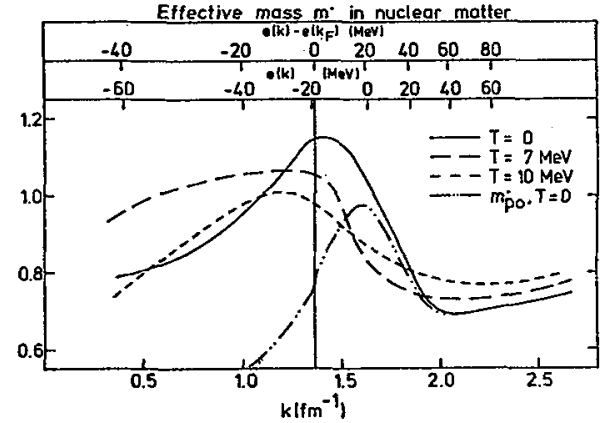

Nucleon effective mass at various temperatures. The curve with double dots and dashes indicates the effective mass calculated with the polarisation potential only.

$$
\text { [F.ig. 4] }
$$
peak in the finite nuclei is perhaps due to the excitation of surface vibrations [11] although the coexistence of the two effects is more probable [12].

As the temperature increases, the peak collapses, but not as quickly as when the polarisation is solely taken into account or as indicated by semi-phenomenological calculations [10]. most important result is the peak appearing around $k_{F}$ at zero temperature. The presence of such a peak in an extended system was surmised on the basis of the calculations up to second order in a semi-phenomenological interaction done in ref. [3] and of extended model calculations for a hard sphere Fermi gas [4] and for a s-wave interaction [5]. The presence of a similar peak in finite nuclei was already conjectured in ref. [6]. It was demonstrated by Mahaux and $\mathrm{Ngô}$ [7] and longly analysed in subsequent works (see ref. [8] for a list of references). It is also predicted by semi-phenomenological calculations

In nuclear matter, the peak appears above $k_{F}$, if only the polarisation contribution is retained (see Fig. 5). The correlation contribution drives the peak to the Fermi level (approximately). Physically, this peak is due to the excita- 


\section{THE MOMENTUM DISTRIBUTION}

An expansion similar to eq. (2.3) exists for the momentum distribution $\rho(k)$, which writes

with

$$
\rho(k)=\rho_{1}(k)+\rho_{2}(k),
$$

and

$$
\begin{aligned}
& \rho_{1}(k)=n(k)\left[2-\bar{m}_{1}\right], \\
& \rho_{2}(k)=(1-n(k)) \bar{m}_{2},
\end{aligned}
$$

$$
\bar{m}_{1}=\left.\frac{\partial}{\partial E} M_{i}(k, E)\right|_{E=e(k)}, \quad i=1,2 \text {. }
$$

The results of our calculation at $T=0$ are given in Fig. 5. The depletion below $\mathrm{k}_{\mathrm{F}}$ is due to the polarisation term and the population above $k_{F}$ is due to the correlation term. One can notice that the depletion of the Fermi sea is quite important. To illustrate this point, we quote that the parameter $x=1-\rho(\bar{k})$, where the bar indicates an average over the Fermi sea, is $\sim 0.25$ in our calculation. In Fig. 6, this value is comcompared with other predictions for $\rho=\rho_{0}$. It turns out that the Paris potential should be classified as a "hard" potential. This property obviously comes from the momentum dependence of the potential.

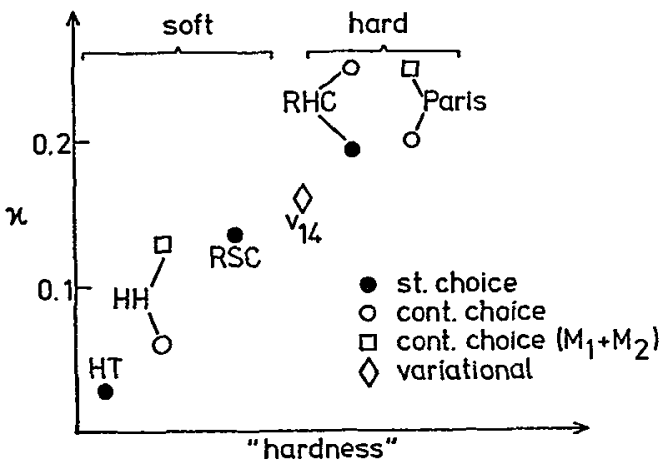

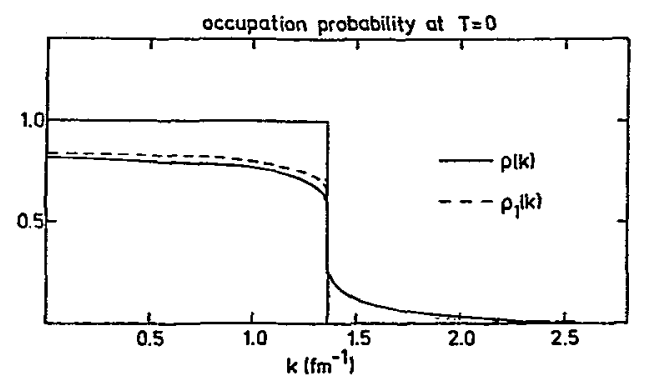

Momentum distribution $\rho(k)$ for cold nuclear matter (full curve). The shortdashed curve represents $\rho_{\uparrow}(k)$ when the self-consistency is applied on the polarisation potential only.

$$
\text { [Fig. 5] }
$$

Illustration of the variation parameter $x$ with the "hardness" of the inner part of various NN potentials : HT (Haftel and Tabakin), HH (Hamman-Ho Kim), RSC (Reid Soft Core), $v_{14}$ (Pandharipande), RHC (Reid Hard Core). The full dots refer to the standard choice of the BruecknerHartree-Fock approximation, the open dots to the continuous choice, the open squares to eqs. (2.3)-(2.6) and the lozenge refers to variational calculations. [Fig. 6]

\section{CONNECTION WITH THE LANDAU-VLASSOV EQUATION}

It is generally believed that the off-equilibrium behaviour of nuclear systems should be governed by a Landau-Vlassov type of equation :

$$
\begin{aligned}
\left(\frac{\partial}{\partial t}+\frac{\vec{k}}{m} \cdot \vec{v}\right) f(\vec{r}, \vec{k}, t)-\frac{1}{h} \sinh \left(k \vec{v} \cdot \vec{v}_{k}(U f(\vec{r}, \vec{k}, t))=\right. \\
\quad \int \frac{d^{3} k_{2}}{(2 \pi)^{3}} \int \frac{d^{3} k_{3}}{(2 \pi)^{3}} \int \frac{d^{3} k_{4}}{(2 \pi)^{4}}\left\{-w\left(\vec{k}_{2}-\vec{k}_{3} \vec{k}_{4}\right) f_{1} f_{2}\left(1-f_{3}\right)\left(1-f_{4}\right)+W\left(\vec{k}_{3} \vec{k}_{4}-\vec{k}_{2}\right) f_{3} f_{4}(1-f)\right. \\
\left.\quad\left(1-f_{2}\right)\right\} \times \delta\left(\vec{k}_{2}+\vec{k}_{2}-\vec{k}_{3}-\vec{k}_{4}\right) \delta\left(e(p)+e\left(p_{2}\right)-e\left(p_{3}\right)-e\left(p_{4}\right)\right)
\end{aligned}
$$


where $f(\vec{r}, \vec{k}, t)$ is the one-body Wigner distribution function and where $f_{i}=$ $f\left(\vec{r}, \vec{k}_{j}, t\right)$. It has been shown recently [13] that both the mean field $U$ and the transition matrix $W$ have to be calculated from Brueckner g-matrix. Of course, in a non-quilibrium process, the g-matrix should be calculated with the instantaneous phase space occupation, but equilibrium calculations can be taken as giving the gross properties of $U$ and $W$

Concerning $U$ we have indicated that this field is nonlocal and depends upon the momentum. In first approximation (in $k$ ), a $\vec{k}$-dependence of the mean field $U$ would introduce the effective mass in the 1.h.s. of eq. (6.1) which then would read

$$
\text { l.h.s. }=\left\{\frac{\partial}{\partial t}+\frac{\vec{k}}{m^{*}} \cdot \vec{\nabla}-(\vec{\nabla} u) \cdot \vec{\nabla}_{k}\right\} f(\vec{r}, \vec{k}, t)
$$

Fig. 4 indicates that this effect is non negligible. It has been studied phenomenoIogically in ref. [14].

As for the structure of the collision term, there is an interesting relationship with the mass operator. Indeed, one can write [1] from eq. (2.3)

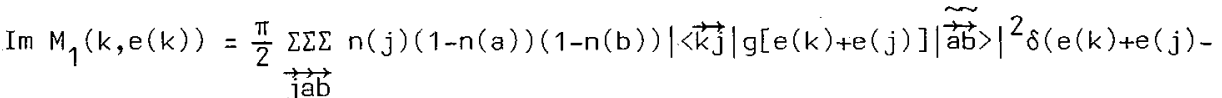

$$
\begin{aligned}
& e(a)-e(b))
\end{aligned}
$$

and from eq. (2.4)

$$
\begin{aligned}
& \operatorname{Im} M_{2}(k, e(k))=-\frac{\pi}{2} \sum \sum \sum n(j) n(l)(1-n(a))|\langle\vec{j} \vec{l}|g[e(j)+e(l)]| \widetilde{\overrightarrow{k a}}\rangle|^{2} \delta(e(k)+e(g)- \\
& \overrightarrow{j \ell a} \\
& e(j)-e(\ell))
\end{aligned}
$$

Assuming that the transition probability $W$ can be identified to the g-matrix [13], one can write the collision term as

$$
\text { r.h.s. }=-\frac{2}{\pi}\left[f \operatorname{Im} M_{1}(k, e(k))+(1-f) \operatorname{Im~} M_{2}(k, e(k))\right]
$$

Strictly speaking, eq. (6.5) holds in a uniform medium, but it is expected to be correct locally in more general situations. The loss term is thus connected to the imaginary part of the polarisation potential and the gain term to the imaginary part of the correlation potential. At equilibrium, one should havé

$$
\frac{\operatorname{Im} M_{2}(k, e(k))}{\operatorname{Im} M_{1}(k, e(k))}=\frac{f}{1-f}
$$

This relation is meaningful for $T \neq 0$ only. For our case, these two ratios are given in Fig. 7 at two temperatures. We recall that $M_{2}$ and $M_{1}$ are calculated independently. Fig. 7 shows that the relation (6.6) is very well fulfilled. The observation that the collision term has the structure (6.5) will help to have a better determination of the input data to be introduced in the collision term. Furthermore, it can be a good starting point for the estimation of equilibrium times and transport properties.

We want to thank C. Mahaux for his interesting comments. 


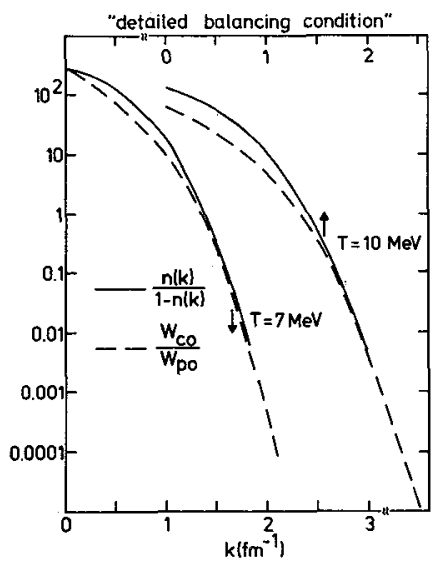

Quantities on the r.h.s. (full curves) and on the l.h.s. (dashed curves) of eq. (6.6)

[Fig. 7]

\section{REFERENCES}

[1] J.-P. Jeukenne, A. Lejeune and C. Mahaux, Phys.Rep. 25C(1976)83

[2] A. Lejeune, P. Grangé, M. Martzolff and J. Cugnon, Nucl. Phys. A453(1986)189

[3] R. Sartor, Nucl.Phys. A267(1976) 29

[4] R. Sartor and C. Mahaux, Phys.Rev. C21(1980)1546

[5] V. Bernard and C. Mahaux, Phys.Rev. C23(1981)888

[6] G.E. Brown, J.H. Gunn and P. Gould, Nucl.Phys. 46(1963) 598

[7] C. Mahaux and H. Ngô, Phys.Lett. 100B(1981) 285

[8] C. Mahaux and R. Sartor, preprint Ljège, February 1987

[9] H. Orland and R. Schaeffer, Nucl.Phys. A299 (1978) 442

[10] R.W. Hasse and P. Schuck, Nucl.Phys. A445(1985) 205

[11] G.F. Bertsch and T.T.S. Kuo, Nucl.Phys. A112 (1968) 204

[12] C. Mahaux, R. Broglia, P.F. Bortignon and C. Dasso, Phys.Rep. 120(1985) 1

[13] W. Botermans and R. Malfliet, Phys.Lett. B171(1986)22

[14] C. Gale, G. Bertsch and S. Das Gupta, UMSI preprint, November 1986. 